\title{
Impact of Intrinsic Motivation Factors; Enjoyment, Autonomy and Impulsiveness on Youth Consumer's Multitasking with Multi-Screens
}

\author{
Shahid Kalim Khan* ${ }^{1}$, Guoxin $\mathrm{Li}^{2}$ and Naqash Ali \\ ${ }^{1,2}$ School of Management, Harbin Institute of Technology, Harbin, China \\ shahid.kalim@hotmail.com
}

\begin{abstract}
Several research opportunities have emerged in many academic disciplines with rise of multiple screens. People now have easy access to a number of display devices like, smartphones, tablets, laptops, TV etc. Most of user's screen time is spent in multitasking with these devices. Multitasking trends are persistent in every age groups but they are most prominent in youth. Despite increasing magnitude of multi-screen multitasking phenomenon, we do not yet have a thorough understanding of individuals' motivations for use of multi-screens in today's complex technology environment. Current study seeks to explore important predictors of the consumer's multi-screen multitasking behavior. Data has been collected from various cities of China through online and offline questionnaire distribution. A total of 344 valid responses have been used for the analysis through Partial Least Square method. Results reveal that, autonomy, enjoyment and impulsiveness as being the intrinsic motivation factor are significant predictors of multi-screen multitasking activities. Research presents valuable implication to industry and literature. Developers and designers should incorporate more hedonistic functions in devices to attract more consumer interest. This study suggests a simple but insightful consumer behavior model based on intrinsic motivation which could be extended to other areas and applications.
\end{abstract}

Keywords: multiscreens; multi-screen; multimedia; multitasking; Media; media multitasking; Chinese consumer's; youth consumers; simultaneous use of multiscreens; digital devices

\section{Introduction}

Multi-Screening is defined as the use of second screen or more than one screens for related activities sequentially and for both related and unrelated activities simultaneously [1]. Before emergence of the multi-screen trend in recent times, user-device relationship has been understood as how users interact with a single device and valuable insights related to consumer's; behavior, attitude and motivation, have been provided by the research in this area which mainly deal with single device interactions [2]. These days, people no more deal with the single device rather they use various devices in sequence or conjunction to extend device possibilities within a broader context. Several research opportunities have emerged due to multi-screen phenomenon in a range of academic disciplines related to user-device relationship.

Media has become diversified with technology innovations over time. Different kinds of devices and contents are available for users to satisfy their various needs [2]. The combinations of multiple devices have resulted in smarter and elevated user experience [3]. Device mobility and power of internet have made multi-screen devices strongly integrate with each other and users are no longer restricted to the single device. They are rather able to choose the right type and appropriate combination of devices which suits their current 
usage needs [2]. Devices such as laptops, smartphones, tablet computers and PDAs are very much integrated these days along with their various software applications and operating systems [3]. Most of our screen time is spent in multitasking with these devices (i.e., simultaneous use). This phenomenon is more dominant in younger consumers as compared to elders [4]. They hold the larger share of the multitasking activities especially in form of media. Presently, screen users are located in such environment where they can do non-stop multitasking, during their travelling, watching television or working on laptop/pc at home or office. Media firms, advertisement agencies, product developers and marketing professionals among others can cultivate larger benefits by better understanding consumers' multi-screen multitasking behavior [4].

Multitasking with computer/software based multi-screen devices is quite common in college and University students within campus or home environment. There are both internal and external reasons for such behavior [5]. Such trends are also related with the affordances provided by the digital devices as currently available computer-based gadgets support and reinforce multitasking. Operating systems on various devices like, laptops, tablets, smart phones and desktop computers are becoming more and more compatible and easier to multitask with them. Now, we can easily handle various related or unrelated tasks within same or different devices. Device "affordance" is the most obvious external reason for multi-screen multitasking. As well as the operating systems on our multi-screen devices such as smart phones, tablets, laptop computers and desktop computers with large monitor screens easily afford multitasking. It is no more difficult to keep many windows open simultaneously both for related or unrelated tasks [6,7]. Importance of emerging multiscreen multitasking phenomenon has vastly been recognized by psychologists and they have tremendously focused on effects of multitasking on people's behavior, health and performance but not many researchers have tried to explore the antecedents of this crucial and affluent consumer behavior aspect [8]. Current study focuses on exploring determinants of consumer's multi-screen multitasking behavior.

\section{Research Framework}

Despite increasing magnitude of multi-screen multitasking phenomenon, we do not yet have a thorough understanding of individuals' motivations for multi-screen use in today's complex environment where they have access to several screens [9]. With substantial research on consumer behavior, not much literature is devoted to answer the question that why they combine screen uses and what are their motivations behind such behavior [10]. A large amount of past research has focused on motivation theories in order to explain information technology adoption and use behavior [11]. Deci [12] categorized these motivations in two broad classes; intrinsic and extrinsic. Intrinsically motivated behaviors happen out of interest without any expectation of separable outcomes, under no external threat, pressure or promise [12]. They are mainly originated by the desirability of interaction with the system itself [13]. When intrinsically motivated, people are interested in the task itself and want to perform it well. In return they get spontaneous experience of satisfaction, interest, excitement, enjoyment and the sense of autonomy associated with performing the activity [14]. On the other hand, expectation of reward, usefulness and external benefits are point of interest in extrinsically motivated behaviors [15].

This research spotlights on intrinsic motivation which is obviously, not the only type of motivation or even volitional activity but, without doubt, it is pervasive and crucial one. Human are active, curious and playful creatures since birth. They have innate ability and desire to learn, explore and they do not necessarily look for external rewards. This natural capability and tendency within themselves is a determining factor in their social, cognitive and physical development. Central to intrinsic motivation is to act out of one's inner interest which really helps one's development of knowledge and skills. Inclination to novelty, innovation, creativeness, autonomy and playfulness, is significant element of 
human nature and contributes considerably towards one's well-being, persistence and performance during their lifetime [16].

Intrinsic motivation consists of spontaneity which means, people tend to act on their impulses in order to get feeling of joy, comfort and excitement [17, 18]. Playfulness or enjoyment is central to intrinsic motivation and most common factor in various intrinsic motivated activities [12]. On the other hand, behaviors which are intrinsically motivated show the prototype of self-determination. Intrinsically motivated people, when performing an interesting task, feel autonomous and volitional and they perceive the task being performed as expression of themselves by them [19]. Figure 1 shown below, represents our proposed research framework.

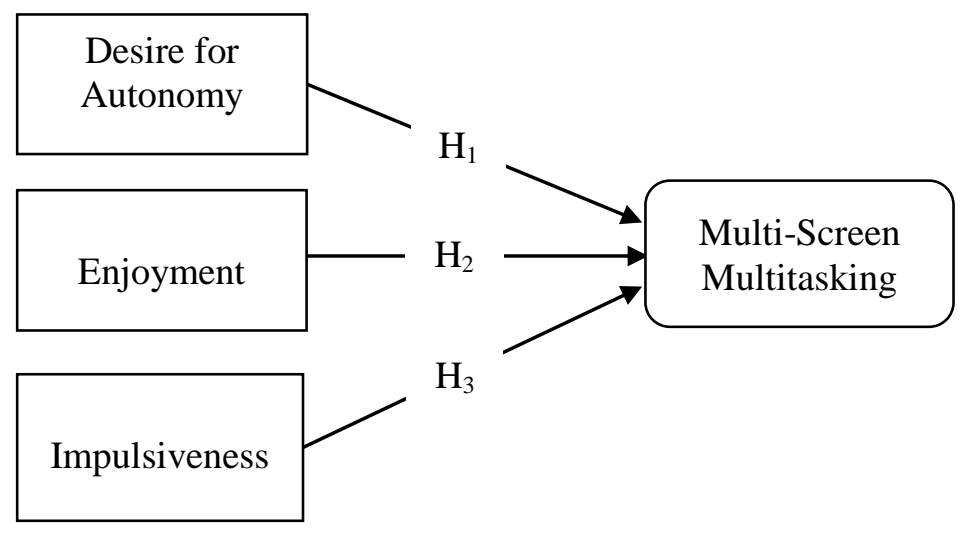

\section{Figure 1. Research Framework}

\subsection{Desire for Autonomy}

Boredom is linked with the lack of autonomy because in the absence of autonomous regulation people are unable to locate and internalize the value of a task [20]. Autonomy is perceived to be consistent with intrinsic motivation as it stems from the self. Human beings have innate desire for autonomy and they desire to sense the experience of control and ability in their own actions [21]. In contrast acting against autonomy and controlling one's behavior because of social or other environmental reasons, can undermine people's intrinsic motivation. Actions lose their appeal when reasons to perform them move from autonomous to control [22].

Autonomous behavior is considered to be self-determined and the one engaging is such behavior feels a sense of choice, enjoyment, interest, satisfaction and personal endorsement [21]. Autonomy is defined as acting out of one's own free will for intrinsic reasons and without any external causes or expectation of extrinsic reward [22]. In context of multiscreen multitasking activities, autonomy can be termed as having the control over choice of what screen and media to be used, in what combination, when to multitask with them and in which situation. In-accordance with above discussion it can be hypothesized that;

H1: Desire for Autonomy associated with use of multi-screens has a positive and significant impact on multi-screens multitasking.

\subsection{Enjoyment}

Enjoyment is defined as "the pleasure individual feels objectively when committing a particular behavior or carrying out a particular activity" [30]. People prefer an activity which is enjoyable as compared to the same activity which is not so enjoyable [31]. In technology oriented environment, enjoyment can be seen as the extent to which using a technology is enjoyable at its own, other than expectation of any extrinsic advantage like performance or efficiency [32]. Previous research has identified that enjoyment pointedly 
influences the use of computers, information technology and software based devices $[33,34,35]$ and it is an important predictor of information system adoption [36,37]. More specifically the hedonic information systems are affected substantially by user's perceived feeling of enjoyment $[38,39]$. It is generally a key factor in determining adoption of any specified system [30,40].

In order to get a pleasurable experience, people seek for sensation on multiple sensory channels [41]. Sensation seeking is linked with multitasking since people higher on sensation seeking behavior multitask more often as compared to low sensation seekers [42]. Also, the high sensation seekers have greater desire for arousal and stimulation. Multitasking can fulfill a person's desire for stimulation by making one engage in multiple activities because it can provide them with more varied and complex experience as compared to use of single medium [8]. For the reasons of fun and enjoyment, multi-screen multitasking activities will be performed more than the activities based on single screen. We can justifiably say that;

$\mathrm{H} 2$ : Enjoyment associated with use of multi-screens has a positive and significant impact on multi-screens multitasking.

\subsection{Impulsiveness}

Some of the choices made by people are driven by impulsiveness in order to achieve short term hedonic interest rather than long than one's long-term benefits [23]. Impulsiveness can broadly be defined as the tendency to act on arising impulses without involving into much thinking [24]. Human behavior can be best understood by combining both impulsive and reflective aspects [25]. In most consumer circumstances, people can experience more or less intense urges to possess or to consume certain products [26]. Through their conscious intentions, they might also abstain from consumption, resist temptations, and adjourn gratification to a later point in time. It should be an important objective for consumer psychologist, marketers, researchers and developers to understand when and why consumer behavior is motivated by impulsivity [25].

Choices driven by impulsiveness originate from the sudden and intense desire of such options [23]. A decision making process is impulsive, when the decision maker selects an option, spontaneously, immediately, unreflectively and kinetically [27]. A decision originated from impulsive-intrinsic motivation is the one that the decision maker had not planned beforehand $[27,28]$. Processing the stimulus in impulsive system triggers three types of feelings. Firstly, it triggers physical senses related to colors or tastes etc. Secondly, it elicits positive and negative effect feelings based on previous or learned experience. Thirdly, it touches cognitive senses like ease of use or familiarity [29]. Use of screen based multimedia is strongly related with all these types of feelings, so, we can conclude from above discussion that impulsiveness is capable of significantly affecting multitasking use behavior of multi-screens. Hence, we can develop the hypothesis as below;

$\mathrm{H} 3$ : Impulsiveness associated with use of multi-screens has a positive and significant impact on multi-screens multitasking.

\section{Methodology}

\subsection{Sampling and Data Collection}

This study is based on empirical data which has been collected through written structured questionnaire. This questionnaire survey has been self-administered and also delivered online through Google docs and Qualdrics.com in several cities of China via email, wechat and personal contacts. Participants are those who have sufficient experience of multi-screen usage. We carefully scrutinized the responses for each question. 14 inappropriate responses such as, having the same answers to all questions or the 
incomplete answers have been excluded from our sample. In total, 344 valid responses are analyzed to check the hypotheses of the research.

\subsection{Measurement Development}

The survey consisted of several questions designed to measure the constructs in our research model and as well as the classification items such as age group, gender, region, education level, frequency of use and types of specific multi-screen devices they are using. Each construct consisted of multiple scale items that were either adapted from existing related studies or self-developed for this study where existing scales were not available. Most of the items have been measured on a five-point Likert scale ranging from 'strongly disagree' to 'strongly agree.' Backward translation (with the questionnaire translated from English into Chinese, and back into English) was used to ensure consistency between the Chinese and the original English version of the instrument.

Face validity of the instrument is established by taking professional advice from a panel of experts and content validity is evaluated by pretesting. Pilot tests have been conducted with a pilot sample of 35 University students. Respondents of the pilot test were requested to provide feedback and suggestions for improvement. Respondents successfully answered all questions with help of the given instructions and they provided some valuable feedback/suggestions. Revised final version of the questionnaire after the pre-testing was distributed for data collection. Table 1 describes all constructs along with their alias used in the analysis model and their respective measurement items for better understanding of the readers.

Table 1. Constructs and Measurement Items

\begin{tabular}{|c|c|c|c|}
\hline Construct & Alias in model & Items & $\begin{array}{l}\text { Source (adapted } \\
\text { from) }\end{array}$ \\
\hline \multirow{8}{*}{$\begin{array}{l}\text { Desire for } \\
\text { Autonomy }\end{array}$} & \multirow{8}{*}{ Autonomy } & $1 \mathrm{~A}$ & {$[47]$} \\
\hline & & $2 \mathrm{~A}$ & {$[48]$} \\
\hline & & $3 \mathrm{~A}$ & \multirow{5}{*}{ [49] } \\
\hline & & $4 \mathrm{~A}$ & \\
\hline & & $5 \mathrm{~A}$ & \\
\hline & & $6 \mathrm{~A}$ & \\
\hline & & $7 \mathrm{~A}$ & \\
\hline & & $8 \mathrm{~A}$ & Self-built \\
\hline \multirow{5}{*}{ Enjoyment } & \multirow{5}{*}{ Enjoy } & $1 \mathrm{D}$ & \multirow{3}{*}{ [50] } \\
\hline & & $2 \mathrm{D}$ & \\
\hline & & $3 \mathrm{D}$ & \\
\hline & & $4 \mathrm{D}$ & \multirow{2}{*}{ Self-developed } \\
\hline & & $5 \mathrm{D}$ & \\
\hline \multirow{6}{*}{ Impulsiveness } & \multirow{6}{*}{ Impulse } & $1 \mathrm{G}$ & \multirow{3}{*}{ [51] } \\
\hline & & $2 \mathrm{G}$ & \\
\hline & & $3 \mathrm{G}$ & \\
\hline & & $4 \mathrm{G}$ & Self-developed \\
\hline & & $5 \mathrm{G}$ & Self-developed \\
\hline & & $6 \mathrm{G}$ & {$[52]$} \\
\hline \multirow{5}{*}{$\begin{array}{l}\text { Multi-screen } \\
\text { Multitasking }\end{array}$} & \multirow{5}{*}{ Multitask } & M1 & \multirow{5}{*}{$\begin{array}{l}\text { Self-developed. Pre- } \\
\text { tested through pilot } \\
\text { study and modified } \\
\text { slightly after pilot } \\
\text { study. }\end{array}$} \\
\hline & & M2 & \\
\hline & & M3 & \\
\hline & & $\begin{array}{l}\text { M4 } \\
\text { M5 }\end{array}$ & \\
\hline & & M6 & \\
\hline
\end{tabular}

3.3. Method for Analyzing the Model 
Structural equation modeling (SEM) through Partial Least Squares (PLS) method has been chosen for the analysis of the data. PLS estimates path models consisting of latent constructs which are indirectly calculated by multiple indicators [38].

\subsection{Respondent's Profile}

Most of our respondents are young and fall into the age range of 18 to 28 years with almost equal percentage of male and female gender. The majority of the respondents are frequent or moderate user of multi-screens which shows that our sample is a good representation of the behavior of interest.

\section{Results and Analysis}

\subsection{Measurement Model}

Reliability and validity of the constructs have been tested by performing composite reliability (CR) and factor loadings as shown in Table 2 and Table 3 respectively.

Table 2. Composite Reliability Coefficients

\begin{tabular}{|c|l|l|l|}
\hline Autonomy & Enjoy & Impulse & Multitask \\
\hline 0.812 & 0.86 & 0.853 & 0.738 \\
\hline
\end{tabular}

The factors loadings are showing satisfactory item reliability. As presented in Table III, all values are higher than 0.679. Normalized factors loading have been used because of the presence of both reflective and formative variables in the model. While composite reliability of the high order construct is at a satisfactory level also, as all indicators are above the 0.7 threshold $(\mathrm{CR}=>0.738)$. These results show adequate discriminant validity for our second order latent variables, because all diagonal values are larger than offdiagonal values in the respective rows and columns as presented in Table 4. Hence, as indicated by these results, our entire constructs meet the criterion for reliability, discriminant validity. Overall, our measurement model is satisfactory so we can proceed with our analysis of the structural model.

Table 3. Normalized Patterns Loadings and Cross-Loadings

\begin{tabular}{|l|l|l|l|l|}
\hline & Autonomy & Enjoy & Impulse & Multitask \\
\hline 1A & $\mathbf{0 . 9 8 1}$ & -0.167 & -0.095 & 0.015 \\
\hline 2A & $\mathbf{0 . 9 8 4}$ & -0.168 & 0.052 & -0.031 \\
\hline 3A & $\mathbf{0 . 9 8}$ & -0.152 & 0.125 & -0.007 \\
\hline 4A & $\mathbf{0 . 9 8 4}$ & -0.112 & -0.032 & 0.135 \\
\hline 5A & $\mathbf{0 . 9 8 4}$ & 0.057 & -0.006 & 0.167 \\
\hline 6A & $\mathbf{0 . 9 6 8}$ & 0.227 & -0.103 & 0.034 \\
\hline 7A & $\mathbf{0 . 9 5 2}$ & 0.128 & 0.09 & -0.264 \\
\hline 8A & $\mathbf{0 . 9 4 5}$ & 0.302 & -0.047 & -0.117 \\
\hline 1D & -0.156 & $\mathbf{0 . 9 7 7}$ & -0.119 & 0.082 \\
\hline 2D & -0.056 & $\mathbf{0 . 9 9 5}$ & -0.079 & -0.004 \\
\hline 3D & -0.051 & $\mathbf{0 . 9 9 6}$ & 0.005 & -0.076 \\
\hline 4D & 0.184 & $\mathbf{0 . 9 2 4}$ & 0.28 & -0.185 \\
\hline 5D & 0.534 & $\mathbf{0 . 7 8 2}$ & 0.218 & 0.236 \\
\hline 1G & -0.08 & -0.013 & $\mathbf{0 . 9 9 6}$ & -0.04 \\
\hline 2G & -0.102 & 0.07 & $\mathbf{0 . 9 9 2}$ & 0.039 \\
\hline 3G & -0.074 & -0.002 & $\mathbf{0 . 9 9 5}$ & -0.069 \\
\hline
\end{tabular}




\begin{tabular}{|l|l|l|l|l|}
\hline 4G & 0.1 & 0.04 & $\mathbf{0 . 9 9 4}$ & 0.036 \\
\hline 5G & 0.207 & -0.167 & $\mathbf{0 . 9 6}$ & 0.085 \\
\hline 6G & 0.092 & -0.016 & $\mathbf{0 . 9 9 6}$ & -0.005 \\
\hline M1 & -0.281 & 0.517 & 0.439 & $\mathbf{0 . 6 7 9}$ \\
\hline M2 & -0.137 & -0.157 & -0.089 & $\mathbf{0 . 9 7 4}$ \\
\hline M3 & 0.187 & 0.053 & 0.133 & $\mathbf{0 . 9 7 2}$ \\
\hline M4 & -0.067 & 0.006 & -0.148 & $\mathbf{0 . 9 8 7}$ \\
\hline M5 & 0.011 & -0.117 & 0.085 & $\mathbf{0 . 9 8 9}$ \\
\hline M6 & 0.196 & -0.098 & -0.204 & $\mathbf{0 . 9 5 4}$ \\
\hline \multicolumn{4}{|l|}{$\begin{array}{l}\text { Note: Loadings and cross-loadings shown are after oblique rotation } \\
\text { and Kaiser Normalization. }\end{array}$}
\end{tabular}

Table 4. Normalized Patterns Loadings and Cross-Loadings

\begin{tabular}{lllll}
\hline & Autonomy & Enjoy & Impulse & Multitask \\
\hline Autonomy & $\mathbf{0 . 5 9 4}$ & & & \\
Enjoy & 0.253 & $\mathbf{0 . 7 4 9}$ & & \\
Impulse & 0.008 & 0.247 & $\mathbf{0 . 7 0 4}$ & \\
Multitask & 0.256 & 0.389 & 0.323 & $\mathbf{0 . 5 6 7}$ \\
\hline
\end{tabular}

\subsection{Structural Model}

The hypotheses have been assessed by examining the parameters provided by the PLS structural model. Table 5 describes the excellent model fit and goodness indices for our PLS structural model.

Table 5. Model Fit and Goodness Indices

\begin{tabular}{|l|l|l|}
\hline Measure & Value & Acceptable Range \\
\hline $\begin{array}{l}\text { Average path coefficient } \\
\text { (APC) }\end{array}$ & $0.262 \mathrm{P}<0.001$ & should be significant \\
\hline Average R-squared (ARS) & $0.273 \mathrm{P}<0.001$ & should be significant \\
\hline $\begin{array}{l}\text { Average adjusted R-squared } \\
\text { (AARS) }\end{array}$ & $0.266 \mathrm{P}<0.001$ & should be significant \\
\hline Average block VIF (AVIF) & 1.092 & $\begin{array}{l}\text { acceptable if }<=5, \text { ideally }<= \\
3.3\end{array}$ \\
\hline $\begin{array}{l}\text { Average full collinearity VIF } \\
\text { (AFVIF) }\end{array}$ & 1.206 & $\begin{array}{l}\text { acceptable if }<=5, \text { ideally }<= \\
3.3\end{array}$ \\
\hline TenenhausGoF (GoF) & 0.344 & $\begin{array}{l}\text { small }>=0.1, \text { medium }>=0.25, \\
\text { large }>=0.36\end{array}$ \\
\hline $\begin{array}{l}\text { R-squared contribution ratio } \\
\text { (RSCR) }\end{array}$ & 1.000 & acceptable if $>=0.9$, ideally $=1$ \\
\hline $\begin{array}{l}\text { Sympson's paradox ratio } \\
\text { (SPR) }\end{array}$ & 1.000 & acceptable if $>=0.7$, ideally $=1$ \\
\hline $\begin{array}{l}\text { Nonlinear bivariate causality } \\
\text { direction ratio }\end{array}$ & 1.000 & acceptable if $>=0.7$ \\
\hline Statistical suppression ratio & 1.000 & acceptable if $>=0.7$ \\
\hline
\end{tabular}




\section{(SSR)}

The results are shown in Figure 2 and Table VI. The $\mathrm{R}^{2}$ value of 0.27 indicates that the theoretical model is explaining a substantial amount of variance in multi-screen multitasking behavior. The theoretical model exhibited substantive explanatory power given the minimum 10 percent criteria which suggests that $R^{2}$ of a dependent variable should be minimum 10 percent to make any meaningful impression. Furthermore, such value of $\mathrm{R}^{2}$ is modest but it is not an unusual phenomenon in social sciences research. There are several studies with low $\mathrm{R}^{2}$ values for example; the well cited study of Cooper and Kleinschmidt [42] consisted of adjusted $\mathrm{R}^{2}$ scores of 0.27 and 0.21 . This value of $\mathrm{R}^{2}$ indicates that there are also some other important factors beyond Autonomy and enjoyment and impulsiveness which can affect multi-screen multitasking behavior.

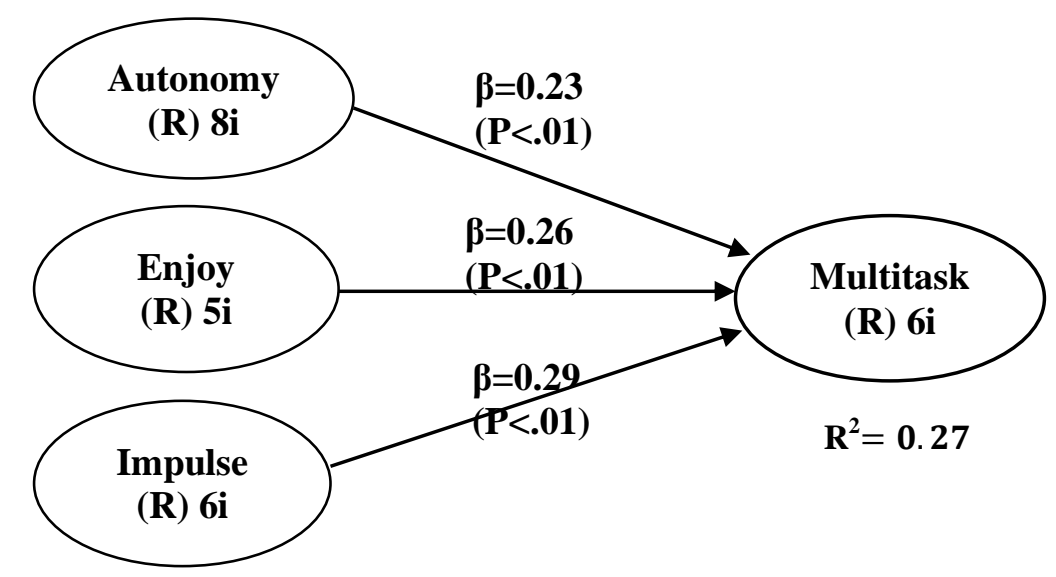

Figure 2. The Structural Model Results.

Table 5. Model Fit and Goodness Indices

\begin{tabular}{|l|l|l|l|}
\hline & Autonomy & Enjoy & Impulse \\
\hline Multitask & 0.233 & 0.262 & 0.291 \\
\hline Hypotheses & $\begin{array}{l}\mathbf{H}_{1} \\
\text { Supported }\end{array}$ & $\begin{array}{l}\mathbf{H}_{2} \\
\text { Supported }\end{array}$ & $\begin{array}{l}\mathbf{H}_{3} \\
\text { Supported }\end{array}$ \\
\hline
\end{tabular}

Figure 2 shows final structural model in which path coefficients of the suggested model can be taken as standardized beta weights, each estimated by controlling all other paths effects. To check if the paths are significant, bootstrapping resampling has been conducted. In bootstrapping resampling, the PLS parameters of a series of random sub samples of the total sample are frequently tested, until significance can be estimated based on their convergent findings. Our structural model can be made an abstract by noting the following significant direct effects of LVs: Autonomy predicted Multitask $(\beta=0.233, p<$ $0.01)$ and Enjoy predicted Multitask $(\beta=0.262, p<0.01)$ while the dependent variable is almost predicting by Impulse about 0.291. Table VI explicitly describes the path coefficients.

Figure 3. presents best fitting curve for multivariate relationship showing interesting curvilinear relationship between Autonomy and Multitask as shown in Figure 3. The line 
is straight when an increase in Autonomy leads to a positive move in Multitasking, indicating a linear relationship. It rises from minimum Autonomy to above average level, implying that the dependent variable increases as the independent variable increases, indicating a positive correlation. Interestingly, the maximum Autonomy is not quiet depicting the maximum Multitask. When Autonomy meets the threshold a negative correlation appears as a recognizable line with a negative slope. As the independent variable increases, the decrease in Multitask is discovered (the line falls as we move to the right from the threshold). The line graph probably shows an important shot for the breathing space of Autonomy and working memory.

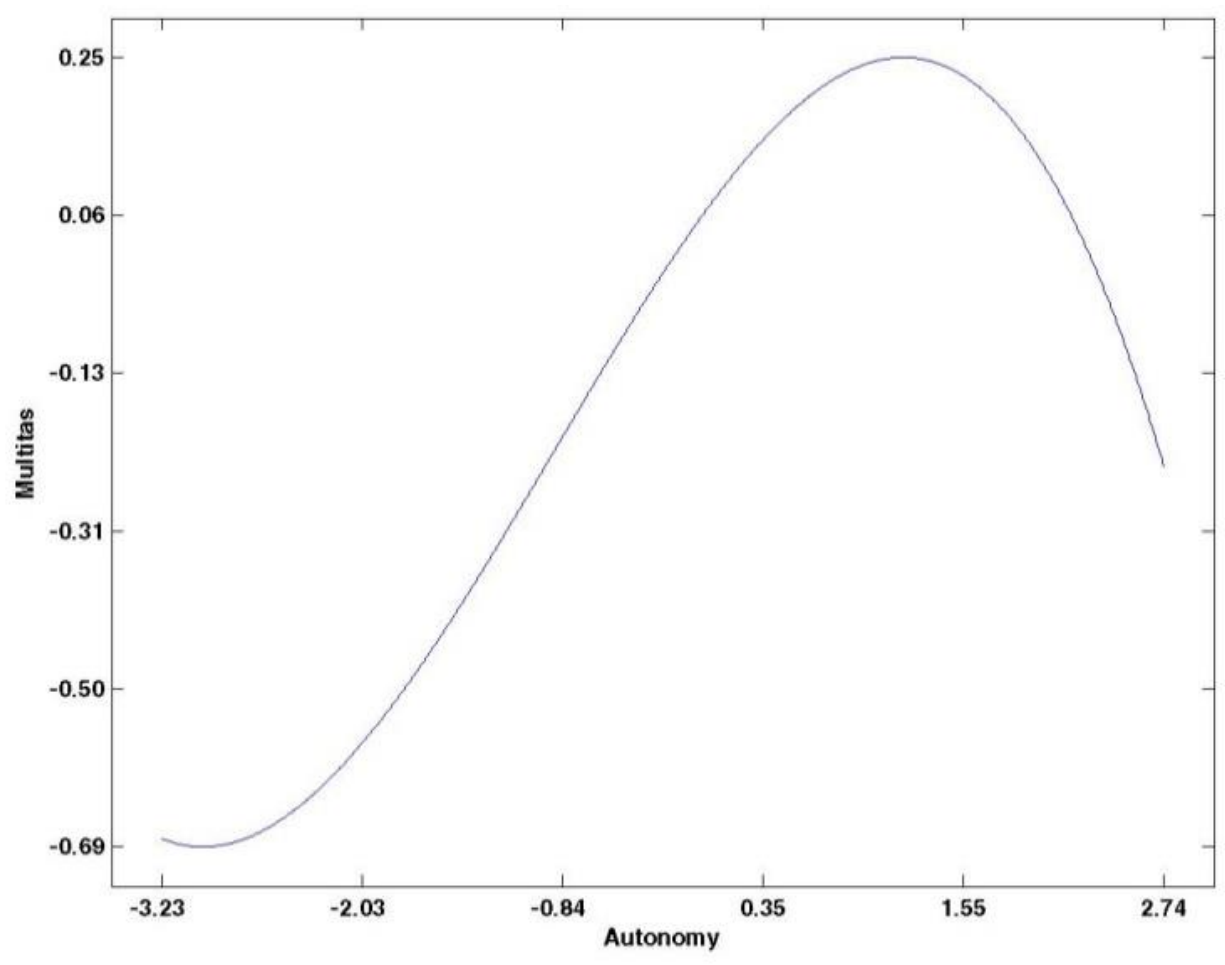

Figure 3. Best fitting Curve for Multivariate Relationship of Autonomy and Multitask

\section{Conclusions and Implications}

This study hypothesizes the three key intrinsic motivation factors as predictors of youth consumers' multi-screen multitasking behavior. Data Analysis supports all three hypotheses and model shows a good fit. Hypothetical model is showing $27 \%$ overall effect on the dependent variable. The theoretical model exhibited substantive explanatory power given the minimum 10 percent criteria which suggests that $R^{2}$ of a dependent variable should be minimum percent to make any meaningful impression. All three constructs have proportionally substantive impact on multi-screen multitasking behavior, however, impulsiveness depicts the highest influence $\left(\mathrm{R}^{2}=0.29\right)$. Which means that people quite often motivated by their impulses when multitasking between screens. While, desire for autonomy and enjoyment are predicting $23 \%$ and $26 \%$ of the consumer multi-screen multitasking behavior.

Desire for Autonomy and impulsiveness have been rarely used in the technology oriented consumer behavior context, this paper makes an important contribution by investigating a modern consumer trend through these vital measures. Overall the current 
research approves the defining role of intrinsic motivation on the multi-screen multitasking phenomenon which holds various practical and academic implications. It has key insights for developers, designers and manufacturers of the multi-screen multimedia devices. They can capture more attraction of the consumers by adding more hedonic content satisfying consumers' intrinsically motivated needs.

The research model introduced by current study may also be applied to a lot of other consumer behavior domains especially in explaining user attitudes towards digital devices, information systems, video/computer games, media multitasking, smart devices and other similar scenarios consisting of hedonic system elements. Motivational factors other than the ones discussed in this study, may be also be tested for the multi-screen multitasking phenomenon. Future research should exploit various research opportunities in various disciplines linked with multi-screens as this area is still quite open for investigations.

\section{Acknowledgments}

This study was supported by funding from the National Natural Science Foundation of China (Grant No. 71272173 and 71490722).

\section{References}

[1] Google, "The New Multi-Screen World Study,"[Online] Google Think Insights, Available at: https://www.thinkwithgoogle.com/research-studies/the-new-multi-screen-world-study.html [Accessed: 25 November 2014], (2012).

[2] I. Liao and Y. Deng, "Understand user-device relationship in multiscreen exercise activity", (2014), pp. $351-362$.

[3] Microsoft Adverstising, Cross screen engagement: Flamingo \& Ipsos OTX, Available at: http://advertising.microsoft.com/international/cl/1932/cross-screen-research-report, (2013), pp. 1-41.

[4] C. K. Coursaris, K. Hassanein, M. M. Head and N. Bontis, "The impact of distractions on the usability and intention to use mobile devices for wireless data services", Comput. Human Behav., vol. 28, no. 4, (2012), pp. 1439-1449.

[5] C. Huang and Y. Kao, "UTAUT2 Based Predictions of Factors Influencing the Technology Acceptance of Phablets by DNP", Math. Probl. Eng., no. 603747, (2015), pp. 1-24.

[6] A. Caras, "What's on other screen [Online]", Microsoft Advertising, Available at: fp.advertising.microsoft.com/.../6. Multi-screen Whitepaper.pdf [Accessed: 10 August 2015], (2010).

[7] H. Baumgartner and J.-B. E.M. Steenkamp, "Exploratory con- sumer buying behavior: conceptualization and measurement", International Journal of Research inMarketing,vol.13, no. 2, (1996), pp. 121-137.

[8] J. Richetin, A. Sengupta and M. Perugini, "A micro-level sim- ulation for the prediction of intention and behavior", Cognitive Systems Research, vol. 11, no. 2, (2010), pp. 181-193.

[9] A. Mansur and T. Kuncoro, "Product inventory predictions at small medium enterprise using market basket analysis approach-neural networks", Procedia Economics and Finance.

[10] D. L. Loudon and A. J. DellaBitta, "ConsumerBehavior:Concepts and Applications", McGraw-Hill, (1993).

[11] D. M. Williams, "Outcome Expectancy and Self-Efficacy: Theoretical Implications of an Unresolved Contradiction", Personal. Soc. Psychol. Rev., vol. 14, no. 4, (2010), pp. 417-425.

[12] N. T. Feather, "Expectations and actions: Expectancy-value models in psychology", (1982).

[13] J. W. Henry, R. W. Stone and W. John, "The effects of computer self-efficacy and outcome expectancy on end-user job control and stress", The effects of computer self-efficacy and outcome expectancy on end-user job control and stress," vol. 8, no. 1, (1999).

[14] D. Potosky, "A field study of computer efficacy beliefs as an outcome of training: The role of computer playfulness", computer knowledge, and performance during training. Computers in Human Behavior, vol. 18 , no. 3 , (2002), pp. 241-255.

[15] B. Hasan, "The influence of specific computer experiences on computer self-efficacy beliefs", Computers in Human Behavior, vol. 19, no. 4, (2003), pp. 443-450.

[16] A. Bandura, "Social foundation of thought and action: A social cognitive theory", New Jersey: Prentice-Hall, (1986).

[17] M. J. Martinko, J. W. Henry and R. W. Zmud, "An attributional explanation of individual resistance to the introduction of information technologies in the workplace", Behaviour \& Information Technology, vol. 15 , no. 5, (1996), pp. 313-330. 
[18] R. W. Stone and J. W. Henry, "The roles of computer self-efficacy and outcome expectancy in influencing the computer end-user's organizational commitment", Journal of End User Computing, vol. 15, no. 1, (2003), pp. 38-53.

[19] F. D. Davis, R. P. Bagozzi and P. R.Warshaw, "User acceptance of computer technology: a comparison of two theoretical models", Manag. Sci., vol. 35, no. 8, (1989), pp. 982-1003.

[20] F. Davis, "Perceived usefulness, perceived ease of use, and user acceptance of information technology", MIS Q., vol. 13, no. 3, (1989), pp. 319-339.

[21] P. S. Ellen, W. O. Bearden and S. Sharma, "Resistance to technological innovations: an examination of the role of self-efficacy and performance satisfaction", Journal of Acad. Mktg Sci., vol.19, (1991), pp. 297-307.

[22] R. Dhar, S. M. Nowlis and S. J. Sherman, "Comparison Construction Effects on Preference”, Journal of Consumer Research, vol. 26, no. 3, (1999), pp.293-306.

[23] I. Simonson," "The effect of product assortment on buyer preferences", Journal of Retailing, vol. 75, no. 3, (1999), pp.347-370. Availableat:mhttp://linkinghub.elsevier.com/retrieve/pii/S0022435999000123 Inpapers3://publication/doi/ 10.1016/S0022-4359(99)00012-3.

[24] D. T. Gilbert, R. B. Giesler and K. A. Morris, "When Comparisons Arise", Journal of Personality and Social cial Psychology, vol. 69, pp. 227-236.

[25] A. Tversky, "Features of Similarity", Psychological Review, (1977), pp. 327-352.

[26] S. P. Mantel and F. R. Kardes, "The Role of Direction of Comparison, Attribute-Based Processing, and Attitude-Based Processing in Consumer Preference", Journal of Consumer Research, (1999), pp. 335352.

[27] D. A. Houston, S. J. Sherman and S. M. Baker, "The Influence of Unique Features and Direction of Comparison on Preferences", Journal of Experimental Social Psychology, (1989), pp. 121-141.

[28] F. R. Kardes and D. M. Sanbonmatsu, "Direction of Comparison, Expected Feature Correlation, and the Set-Size Ef- fect in Preference Judgment", Journal of Consumer Psychol- ogy, vol. 2 no. 1, (1993), pp. 39-54.

[29] R. Dhar and I. Simonson, "The Effect of the Focus of Comparison on Consumer Preferences", Journal of Market- ing Research, (1992), pp. 430-440.

[30] I. Ha, Y. Yoon and M. Choi, "Determinants of adoption of mobile games under mobile broadband wireless access environment”, Information \& Manage- ment, vol. 44, no. 3, (2007), pp. 276-286.

[31] G. S. Rotter and S. M. Portugal, "Group and Individual Effects in Problem Solving", Journal of Applied Psychology vol. 53, no. 4, (1969), pp. 338-341.

[32] M. Henning and A. Jardim, "The Managerial Woman”, Garden City, NY: Anchor Press, (1977).

[33] V. Venkatesh and M. G. Morris, "Why Don't Men Ever Stop to Ask for Directions? Gender, Social Influence, and Their Role in Technology Acceptance and Usage Behavior", MIS Quarterly, vol. 24, no. 1, (2000), pp. 115-139.

[34] J. W. Alba and J. W. Hutchinson, "Dimensions of Con- sumer Expertise", Journal of Consumer Research, vol. 13, no. 4, (1987), pp. 411-454.

[35] F. Sultan, A. J. Rohm and T. Gao, "Factors Influencing Consumer Acceptance of Mobile Marketing: A Two-Country Study of Youth Markets", Journal of Interactive Marketing, vol. 23, no. 4, (2009), pp.308320. Available at: http://dx.doi.org/10.1016/j.intmar.2009.07.003.

[36] R. "Diagnosing measurement equivalence in cross-national research", Journal of International Business Studies, vol. 26, no. 3, (1995), pp. 573-596.

[37] J. Singh, "Measurement issues in cross-national research", Journal of International Business Studies, vol. 26, no. 3, (1995), pp. 597-619.

[38] J. F. Hair, G. T. M. Hult, C. M. Ringle and M. Sarstedt, "A primer on partial least squares structural equation modeling", (PLS-SEM) (1st ed.), London, UK: Sage Publications, (2013).

[39] D. Gefen, D. Straub and M. C. Boudreau, "Structural equation modelling and regression: Guidelines for research practice", Communication of the Association for Information Systems, vol. 4, no. 7, (2000).

[40] W. W. Chin, "The partial least squares approach to structural equation modeling”, In G. A. Marcoulides (Ed.). Modern methods for business research, Lawrence Erlbaum Associates. http://dx.doi.org/10.1016/ j.aap.2008.12.010, vol. 295, (1998), pp. 295-336.

[41] C. Fornell and D. Larcker, "Structural equation models with unobservable variables and measurement error", Journal of Marketing Research, vol. 18, no. 3, (1981), pp. 39-50.

[42] B. Efron, "Bootstrap methods: Another look at the jackknife", Annals of Statistics, vol. 7, (1979), pp. 126.

[43] N. Kock and L. Gaskins, "The Mediating Role of Voice and Accountability in the Relationship Between Internet Diffusion and Government Corruption in Latin America and Sub-Saharan Africa", vol. 20, no. 1, (2014), pp.23-43. 


\section{Authors}
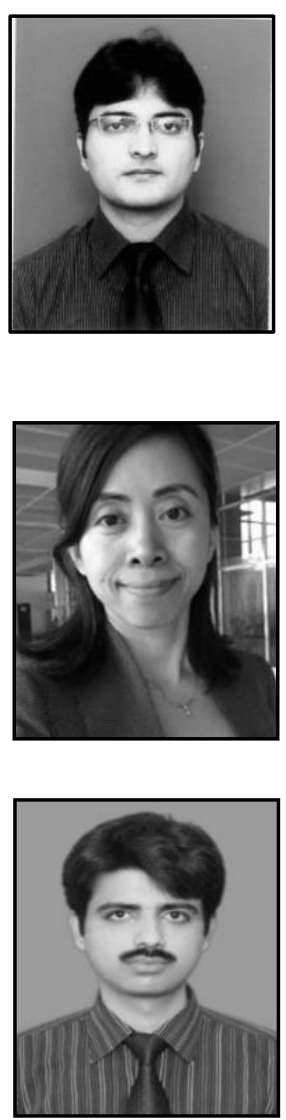

Shahid Kalim Khan is Ph.D candidate at School of Management and Economy at Harbin Institute of Technology, Harbin, China. He earned his Master Degree in Project Management from Royal Institute of Technology (KTH), Sweden. His key research interests are; consumer behaviour, real estate development and marketing, ecommerce and social media. Email: shahid.kalim@hotmail.com, wechat: SK7949

Li Guoxin is a Professor of Marketing and Management at Department of Management and Economy, Harbin Institute of Technology, China. Her key research areas are; social capital and social network, E-commerce, consumer behavior, co-creation and innovation management.

Naqash Ali is graduate student at COMSATS institute of information technology Pakistan. He has earned his MBA degree from the same institution. His recent research interest includes, multiscreen behavior, lagging team members' performance management, brand preference and real estate development in Pakistan. 